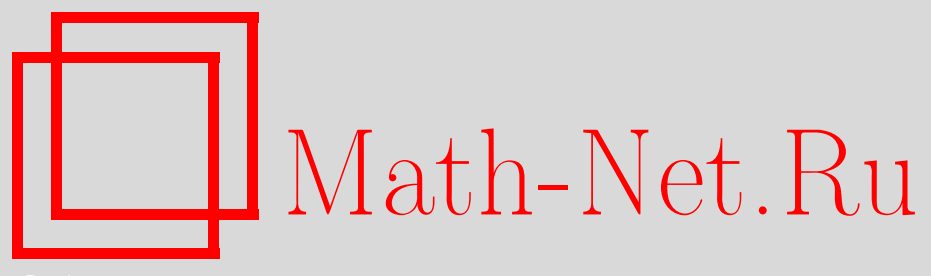

В. А. Головко, Вариационные скобки Схоутена и Нийенхейса, УМH, 2008, том 63, выпуск 2, 165-166

DOI: https://doi.org/10.4213/rm9122

Использование Общероссийского математического портала Math-Net.Ru подразумевает, что вы прочитали и согласны с пользовательским соглашением http://www . mathnet.ru/rus/agreement

Параметры загрузки:

IP : 34.239 .49 .27

26 апреля 2023 г., 05:53:34 


\section{Вариационные скобки Схоутена и Нийенхейса}

\section{В. А. Головко}

Мы показываем, что вариационные скобки Нийенхейса и Схоутена на эволюционных уравнениях можно понимать как суперскобки Якоби на естественных расширениях этих уравнений - так называемых $\ell$ - и $\ell^{*}$-накрытиях. Полученный результат важен для обобщения вариационных скобок на случай произвольных уравнений и нелокальных операторов.

Предварительные сведения (см. [1]). Рассмотрим систему эволюционных уравнений

$$
\frac{\partial u}{\partial t}=f\left(x, t, u, \frac{\partial u}{\partial x}, \ldots, \frac{\partial^{k} u}{\partial x^{k}}\right)
$$

где $u=u(x, t)$ - вектор-функция с компонентами $\left(u^{1}, \ldots, u^{m}\right)$ и $f=\left(f^{1}, \ldots, f^{m}\right)$ также вектор-функция. Пусть $\mathscr{E}$ - бесконечное продолжение системы (1), рассматриваемое как подмногообразие в пространстве джетов $J^{\infty}(\pi)$ тривиального расслоения $\pi: \mathbb{R}^{m} \times \mathbb{R}^{2} \rightarrow \mathbb{R}^{2}$ с координатами $(x, t)$ в базе и $u_{0}^{1}, \ldots, u_{0}^{m}$ вдоль слоя. В качестве координат на $\mathscr{E}$ выбираются функции $x, t$ и $u_{i}^{j}, j=1, \ldots, m, i=0,1,2, \ldots$. Кольцо гладких функций на $\mathscr{E}$ обозначается через $\mathscr{F}(\mathscr{E})$. Основная геометрическая структура на $\mathscr{E}$ - это распределение Картана $\mathscr{C}$, задаваемое полными производными $D_{x}=\frac{\partial}{\partial x}+\sum_{j=1}^{m} \sum_{i \geqslant 0} u_{i+1}^{j} \frac{\partial}{\partial u_{i}^{j}}, \quad D_{t}=\frac{\partial}{\partial t}+\sum_{j=1}^{m} \sum_{i \geqslant 0} D_{x}^{i}\left(f^{j}\right) \frac{\partial}{\partial u_{i}^{j}}$. Симметрии распределения $\mathscr{C}$ образуют алгебру Ли, совпадающую с алгеброй симметрий уравнения (1) и состоящую из эволюционных дифференцирований вида $\vartheta_{\varphi}=\sum_{j=1}^{m} \sum_{k \geqslant 0} D_{x}^{k}\left(\varphi^{j}\right) \frac{\partial}{\partial u_{k}^{j}}$, где вектор-функция $\varphi=\left(\varphi^{1}, \ldots, \varphi^{m}\right)$ удовлетворяет линеаризованному уравнению $\ell_{\mathscr{E}}(\varphi)=0$. Здесь $\ell_{\mathscr{E}}=D_{t}-\sum \frac{\partial f}{\partial u_{i}^{j}} D_{x}^{i}$. Скобка Якоби двух симметрий задается равенством $\{\varphi, \psi\}=\vartheta_{\varphi} \psi-\vartheta_{\psi} \varphi$. Множество вектор-функций $\varphi$ обозначается через $\varkappa$.

Пусть $P$ и $Q-\mathscr{F}(\mathscr{E})$-модули. Операторы $P \rightarrow Q$ в полных производных называются $\mathscr{C}$-дифференциальными. Множество всех таких операторов обозначается через $\mathscr{C} \operatorname{Diff}(P, Q)$. Оператор, сопряженный к $\Delta: P \rightarrow Q$, обозначается через $\Delta^{*}: \widehat{Q} \rightarrow$ $\widehat{P}$, где $\widehat{P}=\operatorname{Hom}_{\mathscr{F}(\mathscr{E})}\left(P, \bar{\Lambda}^{n}\right)$, а $\bar{\Lambda}^{n}-$ пространство горизонтальных дифференциальных $n$-форм, где $n$ - число независимых переменных. Множество $k$-линейных кососимметричных $\mathscr{C}$-дифференциальных операторов $\underbrace{P \times \cdots \times P}_{k \text { раз }} \rightarrow Q$ обозначим через $\mathscr{C} \operatorname{Diff}_{(k)}^{\text {skew }}(P, Q)$, а подмножество в $\mathscr{C} \operatorname{Diff}_{(k)}^{\text {skew }}(P, \widehat{P})$, состоящее из кососопряженных по каждому аргументу операторов, - через $\mathscr{C} \operatorname{Diff}_{(k)}^{\mathrm{sk}-\mathrm{ad}}(P, \widehat{P})$.

Скобка Нийенхейса. Оператор $R \in \mathscr{C} \operatorname{Diff}(\varkappa, \varkappa)$ называется вариационным тензором типа $(1,1)$ на уравнении $(1)$, если

$$
\ell_{\mathscr{E}} \circ R=A \circ \ell_{\mathscr{E}}
$$

для некоторого $\mathscr{C}$-дифференциального оператора $A$. Рассмотрим $\ell$-накрытие уравнения (1), получаемое добавлением к (1) уравнения $q_{t}=\ell_{f}(q)$, где $q$ - новая нечетная зависимая переменная, см. [2].

Работа выполнена при поддержке грантами NWO-РФФИ 047.017.015 и РФФИ-Консорциум Е. I. N. S. T. Е. I. N. 06-01-52060. 
ПРЕДЛОЖЕНИЕ 1. Существует естественное вложение множества вариационных тензоров типа $(1,1)$ в алгебру суперсимметрий $\ell$-накрытия.

Пусть $R_{1}$ и $R_{2}$ - вариационные тензоры типа $(1,1)$. Их вариационной скобкой Нийенхейса называется оператор $\llbracket R_{1}, R_{2} \rrbracket^{\mathrm{N}} \in \mathscr{C} \operatorname{Diff}_{(2)}^{\text {skew }}(\varkappa, \varkappa)$, определяемый равенством

$$
\begin{array}{r}
\llbracket R_{1}, R_{2} \rrbracket^{\mathrm{N}}\left(\varphi_{1}, \varphi_{2}\right)=\left\{R_{1} \varphi_{1}, R_{2} \varphi_{2}\right\}+\left\{R_{2} \varphi_{1}, R_{1} \varphi_{2}\right\}-R_{1}\left(\left\{R_{2} \varphi_{1}, \varphi_{2}\right\}+\left\{\varphi_{1}, R_{2} \varphi_{2}\right\}\right. \\
\left.-R_{2}\left\{\varphi_{1}, \varphi_{2}\right\}\right)-R_{2}\left(\left\{R_{1} \varphi_{1}, \varphi_{2}\right\}+\left\{\varphi_{1}, R_{1} \varphi_{2}\right\}-R_{1}\left\{\varphi_{1}, \varphi_{2}\right\}\right), \quad \varphi_{1}, \varphi_{2} \in \varkappa .
\end{array}
$$

Теорема 1. Имеет место равенство $\mathscr{N}_{\llbracket R_{1}, R_{2} \rrbracket^{\mathrm{N}}}=\left\{\mathscr{N}_{R_{1}}, \mathscr{N}_{R_{2}}\right\}$, где $\mathscr{N}_{R}-$ симметрия, соответствующая вариационному тензору $R$ в силу предложения $1, a\{\varphi, \psi\}=$ $\vartheta_{\varphi} \psi-(-1)^{\bar{\varphi} \cdot \bar{\psi}} \vartheta_{\psi} \varphi-$ суперскобка Якоби на $\ell$-накрытии.

Здесь и ниже $\bar{a}$ обозначает четность элемента $a$.

Скобка Схоутена. Оператор $Q \in \mathscr{C} \operatorname{Diff}^{\text {sk-ad }}(\widehat{\varkappa}, \varkappa)$ называется вариационным бивектором на уравнении (1), если

$$
\ell_{\mathscr{E}} \circ Q=B \circ \ell_{\mathscr{E}}^{*}
$$

для некоторого $\mathscr{C}$-дифференциального оператора $B$. Рассмотрим $\ell^{*}$-накрытие уравнения (1), получаемое добавлением к (1) уравнения $p_{t}=-\ell_{f}^{*}(p)$, где $p-$ новая нечетная зависимая переменная (ср. с [2]).

ПрЕДЛОЖЕНИЕ 2. Существует естественное вложение множества вариационных бивекторов в алгебру суперсимметрий $\ell^{*}$-накрытия.

Пусть $Q_{1}$ и $Q_{2}$ - бивекторы. Их вариационной скобкой Схоутена называется оператор $\llbracket Q_{1}, Q_{2} \rrbracket^{\mathrm{S}} \in \mathscr{C} \operatorname{Diff}_{(2)}^{\text {sk-ad }}(\widehat{\varkappa}, \varkappa)$, определяемый равенством

$$
\begin{array}{r}
\llbracket Q_{1}, Q_{2} \rrbracket^{\mathrm{S}}\left(\psi_{1}, \psi_{2}\right)=-\ell_{Q_{1}, \psi_{1}}\left(Q_{2} \psi_{2}\right)+\ell_{Q_{1}, \psi_{2}}\left(Q_{2} \psi_{1}\right)-Q_{1}\left(\ell_{Q_{2}, \psi_{1}}^{*}\left(\psi_{2}\right)\right) \\
-\ell_{Q_{2}, \psi_{1}}\left(Q_{1} \psi_{2}\right)+\ell_{Q_{2}, \psi_{2}}\left(Q_{1} \psi_{1}\right)-Q_{2}\left(\ell_{Q_{1}, \psi_{1}}^{*}\left(\psi_{2}\right)\right), \quad \psi_{1}, \psi_{2} \in \widehat{\varkappa},
\end{array}
$$

где $\ell_{Q, \psi}(\varphi)=\vartheta_{\varphi}(A)(\psi), \varphi \in \varkappa$.

Теорема 2. Имеет место равенство $\mathscr{H}_{\llbracket Q_{1}, Q_{2} \rrbracket \mathrm{S}}=\left\{\mathscr{H}_{Q_{1}}, \mathscr{H}_{Q_{2}}\right\}$, где $\mathscr{H}_{Q}-$ cuмметрия, соответствующая вариационному бивектору $Q$ в силу предложения 2.

Заключение. Полученные результаты тесно связаны с вопросами интегрируемости нелинейных дифференциальных уравнений. Из уравнения (2) видно, что вариационные тензоры являются операторами рекурсии для симметрий, а уравнение (3) показывает, что если $Q$ - вариационный бивектор, то оператор $Q \circ \delta$, где $\delta$ - оператор Эйлера-Лагранжа, переводит законы сохранения в симметрии. Если при этом $\llbracket Q, Q \rrbracket^{\mathrm{S}}=0$, то $Q$ - гамильтонова структура на рассматриваемом уравнении. Теоремы 1 и 2 позволяют обобщить понятия гамильтоновых структур структур ПуассонаНийенхейса (см. [3]) на произвольные (а не только эволюционные) уравнения и открывают возможности для исследования нелокальных структур подобного рода.

Автор выражает благодарность А. М. Вербовецкому и И. С. Красильщику за обсуждения и ценные советы.

\section{Список литературы}

[1] А.В. Бочаров, В.Н. Четвериков, В.Н. Дужин и др., Симметрии и законы сохранения уравнений математической физики, Факториал, М., 1997. [2] P. Kersten, I. Krasil'shchik, A. Verbovetsky, J. Geom. Phys., 50:1-4 (2004), 273-302; arXiv: math/0304245. [3] Y. Kosmann-Schwarzbach, F. Magri, Ann. Inst. H. Poincaré Phys. Théor., 53:1 (1990), 35-81.

\section{В. А. Головко (V. А. Golovko)}

Московский государственный университет им. М. В. Ломоносова

E-mail: golovko@mccme.ru
Представлено С. К. Ландо Принято редколлегией 09.12.2007 